Iterative 4D cardiac micro-CT image reconstruction using an adaptive spatio-temporal sparsity prior

This article has been downloaded from IOPscience. Please scroll down to see the full text article.

2012 Phys. Med. Biol. 571517

(http://iopscience.iop.org/0031-9155/57/6/1517)

View the table of contents for this issue, or go to the journal homepage for more

Download details:

IP Address: 131.188.201.33

The article was downloaded on 25/03/2013 at 09:51

Please note that terms and conditions apply. 


\title{
Iterative 4D cardiac micro-CT image reconstruction using an adaptive spatio-temporal sparsity prior
}

\author{
Ludwig Ritschl ${ }^{1}$, Stefan Sawall ${ }^{1}$, Michael Knaup ${ }^{1}$, Andreas Hess ${ }^{2}$ \\ and Marc Kachelrie ${ }^{1,3}$ \\ ${ }^{1}$ Institute of Medical Physics (IMP), University of Erlangen-Nürnberg, Henkestrasse. 91, \\ 91052 Erlangen, Germany \\ 2 Institute of Experimental and Clinical Pharmacology and Toxicology, \\ University of Erlangen-Nürnberg, Erlangen, Germany \\ ${ }^{3}$ Medical Physics in Radiology, German Cancer Research Center (DKFZ), \\ Im Neuenheimer Feld 280, 69120 Heidelberg, Germany \\ E-mail: ludwig.ritschl@imp.uni-erlangen.de
}

Received 1 November 2011, in final form 4 January 2012

Published 5 March 2012

Online at stacks.iop.org/PMB/57/1517

\begin{abstract}
Temporal-correlated image reconstruction, also known as 4D CT image reconstruction, is a big challenge in computed tomography. The reasons for incorporating the temporal domain into the reconstruction are motions of the scanned object, which would otherwise lead to motion artifacts. The standard method for 4D CT image reconstruction is extracting single motion phases and reconstructing them separately. These reconstructions can suffer from undersampling artifacts due to the low number of used projections in each phase. There are different iterative methods which try to incorporate some a priori knowledge to compensate for these artifacts. In this paper we want to follow this strategy. The cost function we use is a higher dimensional cost function which accounts for the sparseness of the measured signal in the spatial and temporal directions. This leads to the definition of a higher dimensional total variation. The method is validated using in vivo cardiac micro-CT mouse data. Additionally, we compare the results to phase-correlated reconstructions using the FDK algorithm and a total variation constrained reconstruction, where the total variation term is only defined in the spatial domain. The reconstructed datasets show strong improvements in terms of artifact reduction and lowcontrast resolution compared to other methods. Thereby the temporal resolution of the reconstructed signal is not affected.
\end{abstract}

(Some figures may appear in colour only in the online journal) 


\section{Introduction}

In vivo micro-CT is a very effective imaging modality in preclinical research. It is able to provide three-dimensional volume information of living small animals with a very high spatial resolution. However, there are certain applications which require additional temporal information about the measured animal, for example in cardiac micro-CT. This imaging technique is also known as 4D CT and has been a very active field of research in the last decade.

Due to the periodicity of motions like heartbeat or breathing motion, the standard method to reconstruct temporal-correlated CT volumes is separating these motions into single phases and to reconstruct the corresponding raw data separately. The reconstructed volumes often suffer from undersampling artifacts due to the low number of projections used in each phase. Because low dose exposure is also of high interest in small animal imaging, the number of acquired projections should be held at a low level. One way to handle the problem of missing raw data in each motion phase is the use of iterative reconstruction algorithms which try to incorporate some a priori knowledge into the reconstruction process (Persson et al 2001, Sidky et al 2006, Song et al 2007, Sidky and Pan 2008, Chen et al 2008). In particular, algorithms based on compressed sensing theory (Donoho 2006) show good performance in the presence of undersampled raw data. The optimization problem which is solved by these algorithms is of the following type:

$$
\min \|\Psi(f(\boldsymbol{r}))\|_{1} \text { subject to }\|\mathbf{X} f(\boldsymbol{r})-p\|_{2}^{2}<\epsilon \text {. }
$$

Here, $\mathrm{X}$ is the $\mathrm{X}$-ray transform, $f(\boldsymbol{r})$ is the reconstructed image and $p$ are the measured raw data. The vector $\boldsymbol{r}=(x, y, z)^{T}$ describes the spatial position of a voxel in the reconstructed volume. $\Psi$ is an adequate sparsifying transform of the signal $f(\boldsymbol{r})$. In clinical CT and also in micro-CT, the mostly used transform is the image gradient, which leads to a total variation (TV) prior (Rudin et al 1992). The main assumption is that a medical CT image consists of piecewise constant structures, which makes the image gradient to a good sparsifying transform.

Another way to regularize temporal-correlated image reconstruction is explicitly incorporating the temporal dimension into the reconstruction process (Tian et al 2011, Sawall et al 2011). In this work, we want to follow this strategy and create a sparsifying transform $\psi(\boldsymbol{r}, c)$, which accounts for the higher dimensional properties and periodic temporal behavior of the signal $f(\boldsymbol{r}, c)$. Here, $c$ describes the temporal position, in our special case the cardiac motion phase.

\section{Method}

\subsection{Weighted spatio-temporal TV}

The main innovation of the method presented here is the idea that there exists a high correlation between the images of neighbored motion phases. This can be reflected by the fact that only single parts of the object are moving. This correlation can be used to create a sparsifying transform of the high-dimensional signal $f(\boldsymbol{r}, c)$. Therefore we define, according to the 

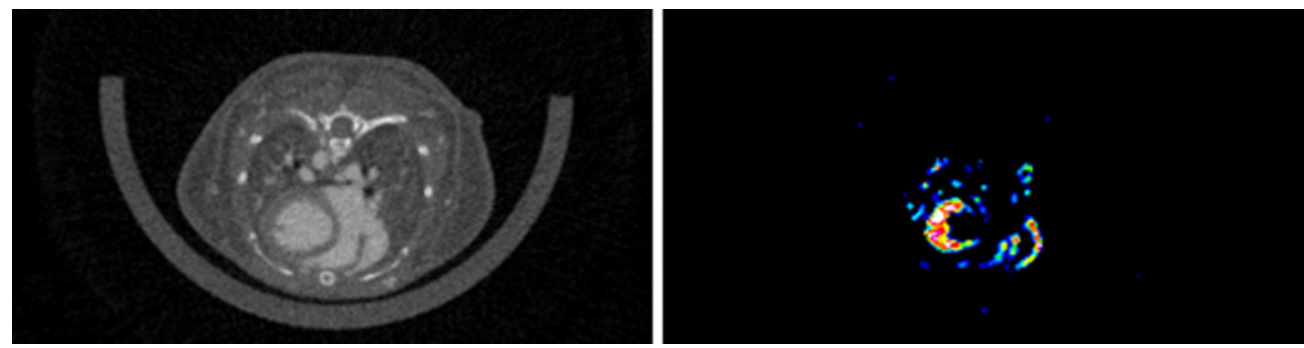

Figure 1. Here the motion map $w(\boldsymbol{r})$ and the corresponding axial slice are shown. Red areas show regions of strong motion. Note the large values of $w(\boldsymbol{r})$ at the borders of the heart.

definition of the spatial TV, an extension of the image gradient to four dimensions:

$$
\nabla_{\boldsymbol{r}, c} f(\boldsymbol{r}, c)=\left(\begin{array}{c}
\frac{1}{\Delta x}\left(f_{x, y, z, c}-f_{x-\Delta x, y, z, c}\right) \\
\frac{1}{\Delta y}\left(f_{x, y, z, c}-f_{x, y-\Delta y, z, c}\right) \\
\frac{1}{\Delta z}\left(f_{x, y, z, c}-f_{x, y, z-\Delta z, c}\right) \\
\frac{1}{\Delta c}\left(f_{x, y, z, c}-f_{x, y, z, c-\Delta c}\right)
\end{array}\right) .
$$

Here, $\Delta x, \Delta y, \Delta z$ and $\Delta c$ represent the spatial or temporal distance of two neighboring voxels. If one of the spatial components involves voxels outside the defined volume, the corresponding term is eliminated. For the temporal components, we assume a cyclic behavior; here $C$ is the number of cardiac phases and $c \in[0, \ldots, 1[$. If the variable $c$ exceeds these boundaries it is returned into this interval by a modulo operation.

Additionally, a spatial adaptive weight function $w(r)$ is introduced. The main idea is that in regions without motion the temporal gradient is the best possible sparsifying transform, whereas regions of high motion can lead to high temporal gradients. Here the spatial sparsity should be enforced instead. The following calculation leads to a temporally averaged motion map of the underlying object:

$$
f_{\text {average }}(\boldsymbol{r})=\mathrm{X}^{-1} p
$$

is the full reconstruction of all acquired projections regardless of the corresponding motion phase. Now the motion map

$$
m(\boldsymbol{r})=\sum_{c} \mathbf{X}_{c}^{-1}\left|\left(\mathbf{X}_{c} f_{\text {average }}(\boldsymbol{r})-p_{c}\right)\right|
$$

consists of higher values in areas of strong motion and lower values in non-moving areas. Here, $\mathrm{X}_{c}$ is the $\mathrm{X}$-ray transform which only treats the projections $p_{c}$ in the corresponding motion phase $c$. Using this information one can create a weight function which should balance the spatial and the temporal gradient. In this study we used

$$
w(\boldsymbol{r})=\frac{m(\boldsymbol{r})-m_{\min }}{m_{\max }-m_{\min }}, \text { with } m_{\max }=\max (m(\boldsymbol{r})) \text { and } m_{\min }=\min (m(\boldsymbol{r})) .
$$

Figure 1 shows this motion map and the corresponding slice of the volume. 

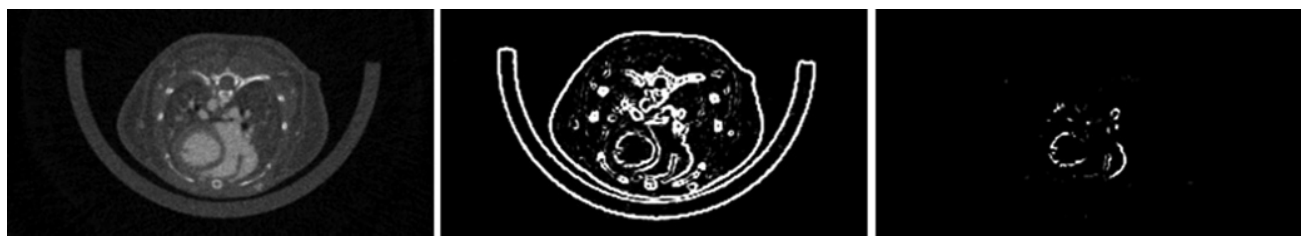

Figure 2. The left image shows one axial slice of the reconstructed volume at one motion phase. The middle image shows the absolute values of the spatial gradient. In the right image one can see the absolute values of the weighted four-dimensional gradient of the corresponding slice. Note the sparsity of the right image. The middle and the right images are shown at the same gray level.

Using this function $w(\boldsymbol{r})$ one can create the diagonal matrix

$$
\boldsymbol{W}(\boldsymbol{r})=\left(\begin{array}{cccc}
w^{2}(\boldsymbol{r}) & 0 & 0 & 0 \\
0 & w^{2}(\boldsymbol{r}) & 0 & 0 \\
0 & 0 & w^{2}(\boldsymbol{r}) & 0 \\
0 & 0 & 0 & (1-w(\boldsymbol{r}))^{2}
\end{array}\right)
$$

Now the weighted high-dimensional total variation (HDTV) is defined as

$$
\begin{aligned}
\operatorname{HDTV} f(\boldsymbol{r}, c) & =\left\|\nabla_{\boldsymbol{r}, c} f(\boldsymbol{r}, c)\right\|_{1}^{W} \\
& =\sum_{\boldsymbol{r}, c} \sqrt{\left(\nabla_{\boldsymbol{r}, c} f(\boldsymbol{r}, c)\right)^{T} \boldsymbol{W}(\boldsymbol{r}) \nabla_{\boldsymbol{r}, c} f(\boldsymbol{r}, c) .}
\end{aligned}
$$

In figure 2, one can see the absolute values of the spatial gradient transform and the weighted HDTV transform.

The minimization problem which has to be solved now for image reconstruction is

$$
\begin{array}{r}
\min \left\|\nabla_{\boldsymbol{r}, c} f(\boldsymbol{r}, c)\right\|_{1}^{W}, \text { subject to } \\
\sum_{c}\left\|\mathbf{X}_{c} f(\boldsymbol{r}, c)-p_{c}\right\|_{2}^{2}<\epsilon .
\end{array}
$$

\subsection{Minimization algorithm}

To find a solution of equation (2) an approximative method, which has been developed for TV-constrained image reconstruction, is used. The single steps are shortly explained here; for a more detailed description we refer the reader to Ritschl et al (2011).

To minimize the cost functional the two terms of equation (2) are treated separately. The raw data fidelity term is minimized by SART (Andersen and Kak 1984), which performs update steps in the form of

$$
f_{n+1}(\boldsymbol{r}, c)=f_{n}(\boldsymbol{r}, c)+\beta \frac{1}{\mathrm{X}_{c}^{\mathrm{T}} 1} \mathbf{X}_{c}^{\mathrm{T}} \frac{\mathrm{X}_{c} f_{n}(\boldsymbol{r}, c)-p_{c}}{\mathrm{X}_{c} 1} .
$$

The index $n$ represents the iteration number. In the following, the index $n$ will represent a so-called outer iteration. The relaxation parameter $\beta$ has been set to $\beta=0.8$.

After applying this SART update to all phases $c$, the gradient descent method is applied to $f_{n+1}(\boldsymbol{r}, c)^{\mathrm{SART}}$ to minimize the TV. An iteration of the gradient descent is given by

$$
f_{n+1, m+1}^{\mathrm{TV}}(\boldsymbol{r}, c)=f_{n+1, m}^{\mathrm{TV}}(\boldsymbol{r}, c)-\alpha \cdot \operatorname{grad}\left\|\nabla_{\boldsymbol{r}, c} f_{n+1, m}^{\mathrm{TV}}(\boldsymbol{r}, c)\right\|_{1} .
$$

As an initialization input $f_{n+1,0}^{\mathrm{TV}}(\boldsymbol{r}, c)$, we use $f_{n+1}^{\mathrm{SART}}(\boldsymbol{r}, c) . m$ is the index of the gradient descent iteration, which is the inner iteration. In this case we used $M=20$ gradient descent steps. 
The parameter $\alpha$ is calculated individually in each update step by a backtracking linesearch to ensure a minimization of the HDTV (Boyd and Vandenberghe 2004).

The linear combination

$$
f_{n+1}(\boldsymbol{r}, c, \lambda)=(1-\lambda) f_{n+1}^{\mathrm{SART}}(\boldsymbol{r}, c)+\lambda f_{n+1, M}^{\mathrm{TV}}(\boldsymbol{r}, c)
$$

is the final update image of one iteration. The weight $\lambda$ is determined analytically by solving

$$
\sum_{c}\left\|\mathbf{X}_{c} f_{n+1}(\boldsymbol{r}, c, \lambda)-p_{c}\right\|_{2}^{2}=(1-\omega) \epsilon_{n+1}^{\mathrm{SART}}+\omega \epsilon_{n}
$$

with

$$
\epsilon_{n+1}^{\mathrm{SART}}=\sum_{c}\left\|\mathrm{X}_{c} f_{n+1}^{\mathrm{SART}}(\boldsymbol{r}, c)-p_{c}\right\|_{2}^{2}
$$

and

$$
\epsilon_{n}=\sum_{c}\left\|\mathbf{X}_{c} f_{n}(\boldsymbol{r}, c)-p_{c}\right\|_{2}^{2}
$$

$\omega$ is a user-defined parameter; here we set it to $\omega=0.8$. As a stopping criterion

$$
\text { stop if } \Delta \epsilon_{n+1}^{\mathrm{SART}}<\Delta \epsilon_{\mathrm{lim}}
$$

with

$$
\Delta \epsilon_{\lim }=0.01 \cdot\left(\epsilon_{1}-\epsilon_{2}\right)
$$

was used.

\section{Results}

\subsection{Data acquisition and gating}

For the evaluation of the method an in vivo scan of a mouse has been acquired. The data were measured with a dedicated in vivo conebeam micro-CT scanner installed at the Institute of Medical Physics, Erlangen, Germany. The mouse was anesthetized with a combination of ketamine and rompun. A blood pool contrast agent (Binition, Binitio Biomedical Inc., Ottawa) was used for contrast enhancement. The scans were conducted at $65 \mathrm{kV}$ tube voltage, and the tube current time product was $86.4 \mathrm{~mA}$ s. The number of acquired projections is 7200 . The integration time of the detector is $40 \mathrm{~ms}$ per projection. These projections were acquired within five rotations during a time interval of $288 \mathrm{~s}$. The average heartbeat of a mouse is about 450 beats per minute, and the average number of breathing cycles is about 300 per minute.

Due to the fact that there are two combined motions present, the breathing and the heart motion, a combined motion estimation has been done. Therefore, a kymogram-based motion detection method (Kachelrieß et al 2002) has been applied to the raw data. The corresponding phase information was created using a retrospective gating. Both motions were divided into ten equally spaced motion phases. In this study only the heart motion was considered, which means we used only the projections from one breathing phase. This leads to an effectively used number of 702 projections, which cover all ten heart phases. The number of projections in each motion phase $c$ is between 55 and 95. The sampling width of the variable $c$ is $\Delta c=0.1$.

To pre-correct the data for scatter and beamhardening, the empirical cupping correction (Kachelrieß et al 2006) method was applied before reconstruction. 

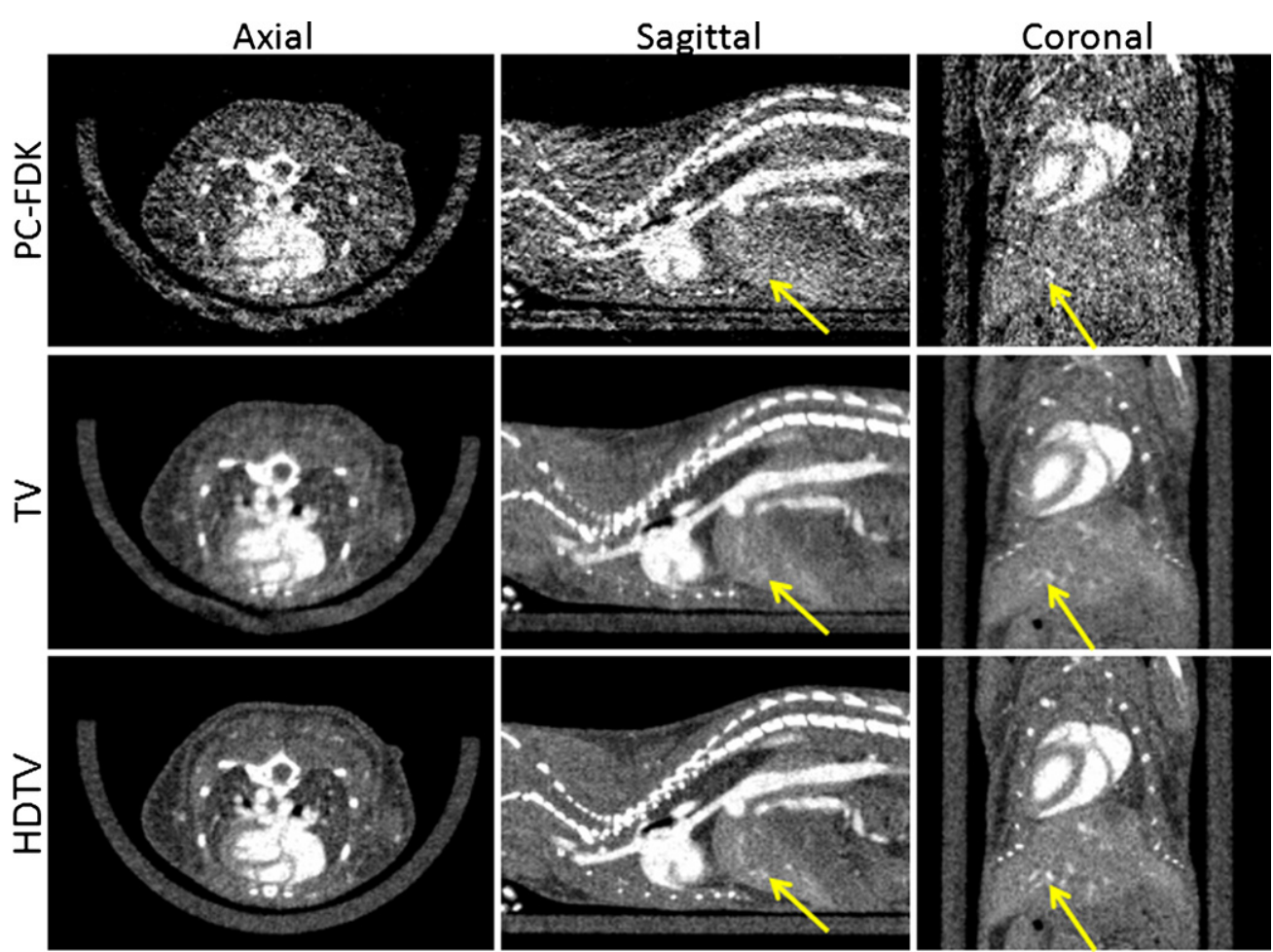

Figure 3. This image shows the results of the different reconstruction algorithms. For all algorithms axial, sagittal and coronal views are shown. Note the improved visibility of the small vessels in the liver for the HDTV case. All images are windowed $(C=100 \mathrm{HU}, W=1200 \mathrm{HU})$.

\subsection{Reconstruction of the acquired data}

The dataset was reconstructed using three different methods. The first is the FDK (Feldkamp et al 1984) algorithm, which reconstructs the raw data $p_{c}$ of each phase separately. In the following it will be denoted with phase-correlated FDK (PC-FDK). The second one is an iterative TV-based algorithm (Ritschl et al 2011), which minimizes equation (1) with $\Psi(\boldsymbol{r})=\nabla_{\boldsymbol{r}}$. The third is the HDTV method proposed in this paper.

The size of the volume is $(45 \times 45 \times 56.25) \mathrm{mm}^{3}$. The number of voxels is $512^{3}$. Figures 3 and 4 show the results of the three algorithms at two different window settings.

In all figures, the TV and the HDTV reconstructions are shown at the same level of raw data consistency to enable a fair comparison. The number of iterations for the TV case was between 18 and 23 iterations; here every motion phase has been reconstructed separately. For the HDTV reconstruction 20 iterations were performed.

\section{Discussion}

\subsection{Spatial resolution and soft tissue contrast}

Regarding figure 3 one can see that both iterative methods lead to a strong improvement in terms of streak artifact reduction and low-contrast information compared to the PC-FDK reconstructions. The high-dimensional TV image shows better low-contrast characteristics 

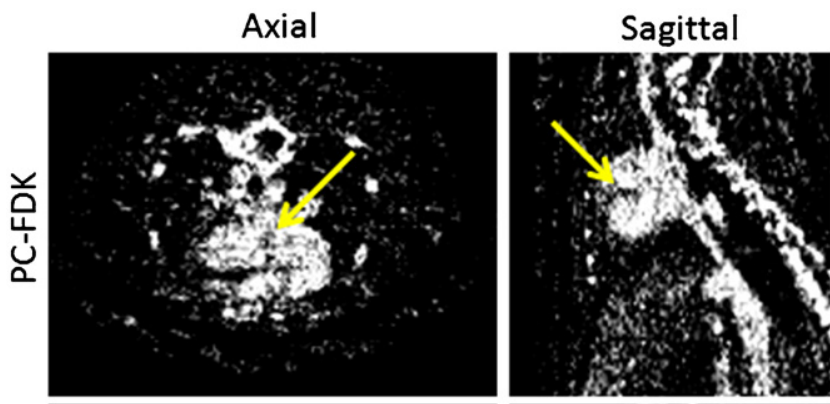

\section{Coronal}


Figure 4. Here the results of the different reconstruction algorithms are shown. The images focuses on the contrast-enhanced heart region. The HDTV reconstructions show a strong improvement in terms of spatial resolution. For all algorithms axial, sagittal and coronal views are shown. All images are windowed $(C=400 \mathrm{HU}, W=700 \mathrm{HU})$.

compared to the pure spatial TV-based reconstruction. The gaps in the acquired projection data lead to artifacts, which are still present in the spatial TV images. The additional information incorporated by the temporal regularization is able to compensate for them. In particular, the small vessels in the liver, marked by the arrows, are only clearly visible in the HDTV reconstruction.

Figure 4 focuses on the heart region. Here it is easy to see that the HDTV reconstruction is superior in terms of spatial resolution and contrast. Also here the spatial TV leads to a significant improvement relative to the FDK reconstruction, but it is not able to recover detailed structures inside the heart.

\subsection{Temporal resolution}

The preservation of the temporal resolution by the HDTV method is an important aspect. Figures 5 and 6 show some evaluations concerning this aspect. Figure 5 shows an FDK reconstruction of all projections regardless of their corresponding heart motion phase, which means $\Delta c=1.0$. It can also be seen as an averaged image of all ten PC-FDK reconstructions 

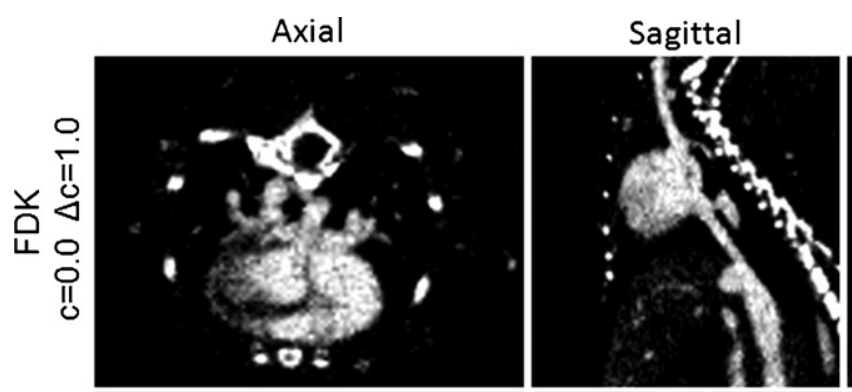

Coronal
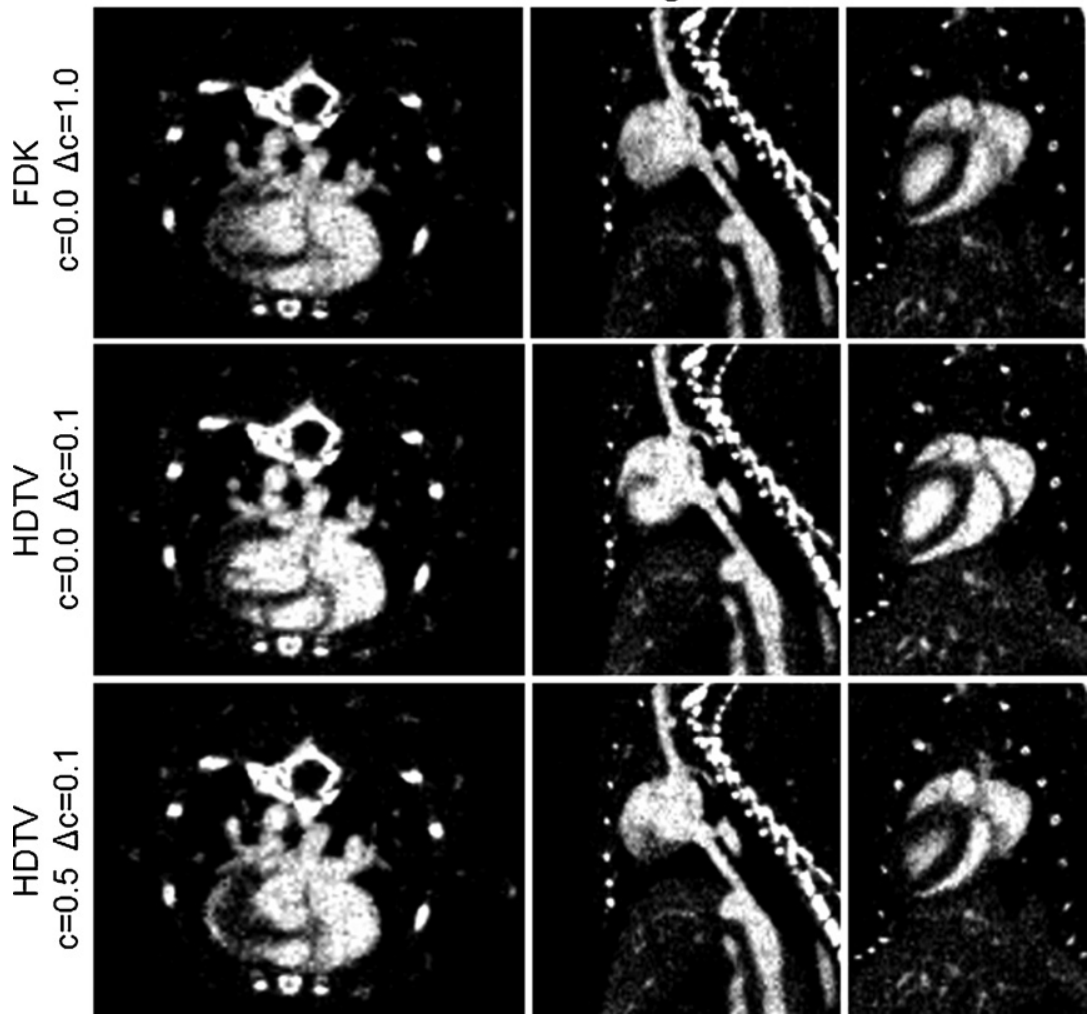

Figure 5. The first row shows a temporally averaged FDK reconstruction without any temporal resolution. The two rows below show the HDTV reconstructions of different motion phases $c$. The less blurry HDTV images indicate a preservation of the temporal resolution. All images are windowed $(C=400 \mathrm{HU}, W=700 \mathrm{HU})$.
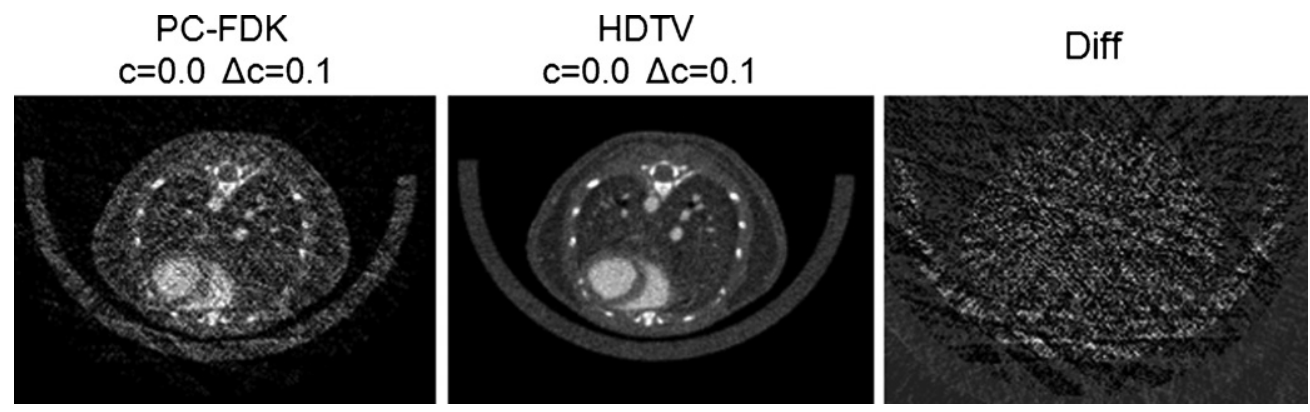

Figure 6. Here the FDK and the HDTV reconstruction and the difference image between them are shown. As one can see the difference, there is no loss of temporal resolution. The FDK and the HDTV images are windowed $(C=0 \mathrm{HU}, W=1000 \mathrm{HU})$, and also the difference image is windowed $(C=0 \mathrm{HU}, W=200 \mathrm{HU})$.

with $\Delta c=0.1$. It shows a low noise and streak artifact level but has no temporal resolution. Additionally, HDTV reconstructions at $c=0.0$ and $c=0.5$ with $\Delta c=0.1$ are shown. They show different positions of the heart relative to each other and are less blurry in the heart region than the averaged FDK image. Figure 6 shows an axial slice at the motion phase $c=0.0$ of the 
PC-FDK and the HDTV image and their difference image. The fact that there is no anatomical structure of the heart visible in the difference image confirms that the temporal resolution of the HDTV reconstructions is preserved

\section{Conclusion}

We presented a new method for spatial and temporal regularization in the context of temporalcorrelated CT image reconstruction. Therefore, the weighted higher dimensional total variation has been defined. The proposed algorithm was evaluated using in vivo micro-CT mouse data. Using the proposed cost function leads to a much better artifact reduction and higher lowcontrast information compared to other methods. Thereby the temporal resolution of the signal is preserved. Applying this method leads to a strong dose reduction because it enables a strong undersampling of projection views. An extension to higher dimensional problems as proposed in Sawall et al (2011) can be done in a straightforward way.

\section{Acknowledgments}

This study was supported in parts by the Deutsche Forschungsgemeinschaft (DFG) under grant no. FOR 661 and by Ziehm Imaging GmbH, Nürnberg, Germany. The high-speed image reconstruction software RayConStruct-IR was provided by RayConStruct GmbH, Nürnberg, Germany. We thank Sandra Strobelt for carrying out the mouse measurements. Additionally, we thank the Intel Corporation and the Fujitsu Group for providing their latest multicore hardware.

\section{References}

Andersen A H and Kak A C 1984 Simultaneous algebraic reconstruction technique (SART): a superior implementation of the ART algorithm Ultrason. Imaging 6 81-94

Boyd S and Vandenberghe L 2004 Convex Optimization (Cambridge: Cambridge University Press)

Chen G-H, Tang J and Leng S 2008 Prior image constrained compressed sensing (PICCS): a method to accurately reconstruct dynamic CT images from highly undersampled projection data sets Med. Phys. 35 660-3

Donoho D L 2006 Compressed sensing IEEE Trans. Inform. Theory 52 1289-306

Feldkamp L, Davis L and Kress J 1984 Practical conebeam algorithm J. Opt. Soc. Am. 1 612-9

Kachelrieß M, Sennst D A, Maxlmoser W and Kalender W A 2002 Kymogram detection and kymogram-correlated image reconstruction from subsecond spiral computed tomography scans of the heart Med. Phys. 29 1489-503

Kachelrieß M, Sourbelle K and Kalender W 2006 Empirical cupping correction: a first order raw data precorrection for cone-beam computed tomography Med. Phys. 33 1269-74

Persson M, Bone D and Elmquist H 2001 Total variation norm for three-dimensional iterative reconstruction in limited view angle tomography Phys. Med. Biol. 28 853-66

Ritschl L, Bergner F, Fleischmann C and Kachelrieß M 2011 Improved total variation-based CT image reconstruction applied to clinical data Phys. Med. Biol. 56 1545-61

Rudin L, Osher S and Fatemi E 1992 Nonlinear total variation based noise removal algorithms J. Phys. D: Appl. Phys. 60 259-68

Sawall S, Bergner F, Karolczak M, Kachelrieß M, Lapp R, Mronz M and Hess A 2011 Low-dose cardio-respiratory phase-correlated cone-beam micro-CT of small animals Med. Phys. 38 1416-24

Sidky E Y, Kao C and Pan X 2006 Accurate image reconstruction from few-views and limited-angle data in divergentbeam CT J. X-Ray Sci. Technol. 14 119-39

Sidky E Y and Pan X 2008 Image reconstruction in circular cone-beam computed tomography by total variation minimization Phys. Med. Biol. 53 4777-807

Song J, Liu Q H, Johnson G A and Badea C T 2007 Sparseness prior based iterative image reconstruction for retrospectively gated cardiac micro-CT Med. Phys. 34 4476-83

Tian Z, Jia X, Dong B, Lou Y and Jiang S B 2011 Low-dose 4DCT reconstruction via temporal nonlocal means Med. Phys. 38 1359-65 